\title{
Actionable gene-based classification toward precision medicine in gastric cancer
}

\author{
Hiroshi Ichikawa ${ }^{1 *}$ DD, Masayuki Nagahashi', Yoshifumi Shimada', Takaaki Hanyu', Takashi Ishikawa', \\ Hitoshi Kameyama', Takashi Kobayashi', Jun Sakata', Hiroshi Yabusaki², Satoru Nakagawa², Nobuaki Sato ${ }^{3}$, \\ Yuki Hirata ${ }^{4}$, Yuko Kitagawa ${ }^{4}$, Toshiyuki Tanahashi ${ }^{5}$, Kazuhiro Yoshida ${ }^{5}$, Ryota Nakanishi ${ }^{6}$, Eiji Oki ${ }^{6}$, Dana Vuzman ${ }^{7,8}$, \\ Stephen Lyle ${ }^{9 *}$, Kazuaki Takabe ${ }^{10,11}$, Yiwei Ling ${ }^{12}$, Shujiro Okuda ${ }^{12}$, Kohei Akazawa ${ }^{13}$ and Toshifumi Wakai ${ }^{{ }^{*}}$
}

\begin{abstract}
Background: Intertumoral heterogeneity represents a significant hurdle to identifying optimized targeted therapies in gastric cancer (GC). To realize precision medicine for GC patients, an actionable gene alteration-based molecular classification that directly associates GCs with targeted therapies is needed.

Methods: A total of 207 Japanese patients with GC were included in this study. Formalin-fixed, paraffin-embedded (FFPE) tumor tissues were obtained from surgical or biopsy specimens and were subjected to DNA extraction. We generated comprehensive genomic profiling data using a 435-gene panel including 69 actionable genes paired with US Food and Drug Administration-approved targeted therapies, and the evaluation of Epstein-Barr virus (EBV) infection and microsatellite instability (MSI) status.

Results: Comprehensive genomic sequencing detected at least one alteration of 435 cancer-related genes in 194 GCs (93.7\%) and of 69 actionable genes in 141 GCs (68.1\%). We classified the 207 GCs into four The Cancer Genome Atlas (TCGA) subtypes using the genomic profiling data; EBV $(N=9)$, MSI $(N=17)$, chromosomal instability $(N=119)$, and genomically stable subtype $(N=62)$. Actionable gene alterations were not specific and were widely observed throughout all TCGA subtypes. To discover a novel classification which more precisely selects candidates for targeted therapies, 207 GCs were classified using hypermutated phenotype and the mutation profile of 69 actionable genes. We identified a hypermutated group $(N=32)$, while the others $(N=175)$ were sub-divided into six clusters including five with actionable gene alterations: ERBB2 $(N=25), C D K N 2 A$, and CDKN2B $(N=10), \operatorname{KRAS}(N=10), B R C A 2(N=9)$, and ATM cluster $(N=12)$. The clinical utility of this classification was demonstrated by a case of unresectable GC with a remarkable response to anti-HER2 therapy in the ERBB2 cluster.
\end{abstract}

Conclusions: This actionable gene-based classification creates a framework for further studies for realizing precision medicine in GC.

Keywords: Gastric cancer, Next-generation sequencing, Gene panel, Precision medicine, Actionable gene

\footnotetext{
* Correspondence: hichikawa-nii@med.niigata-u.ac.jp;

Stephen.lyle@umassmed.edu; wakait@med.niigata-u.ac.jp

1Division of Digestive and General Surgery, Niigata University Graduate

School of Medical and Dental Sciences, 1-757 Asahimachi-dori, Chuo-ku,

Niigata City, Niigata 951-8510, Japan

${ }^{9}$ Molecular, Cell \& Cancer Biology, University of Massachusetts Medical

School, 55 Lake Avenue North, Worcester, Massachusetts 01655, USA

Full list of author information is available at the end of the article
} 


\section{Background}

Gastric cancer (GC) is the third leading cause of cancer death worldwide, with the highest incidence in Eastern Asia [1]. Despite the recent advances in cytotoxic chemotherapy, unresectable or recurrent GC remains notorious for the dismal prognosis of patients, with median survival ranging from 13.0 to 16.6 months [2, 3]. A number of clinical trials of targeted therapies have been conducted to improve the outcome of unresectable or recurrent GC [4]. To date, only two drugs have demonstrated significant efficacy: trastuzumab, an anti-HER2 antibody [5], and ramucirumab, an anti-VEGF receptor 2 antibody [6, 7]. The other targeted therapies failed to show any survival benefit in phase III clinical trials, deemed most likely due to the heterogeneous nature of GC and the lack of appropriate molecular biomarkers. Therefore, a better understanding of the molecular profiles of GC and optimum patient selection based on the molecular alterations is necessary to facilitate targeted therapies for $\mathrm{GC}$.

Large-scale comprehensive molecular profiling using next-generation sequencing (NGS) technologies has identified the molecular landscape of a number of cancers, including GC [8-13]. The Cancer Genome Atlas (TCGA) cohort classified heterogeneous GC into four molecular subtypes: Epstein-Barr virus positive tumors (EBV), tumors with microsatellite instability (MSI), tumors with chromosomal instability (CIN), and genomically stable tumors (GS) based on various technologies, including whole-exome sequencing (WES) [10]. On the other hand, the Asian Cancer Research Group (ACRG), using array-based gene expression profiling, identified four distinct molecular subtypes that were associated with patient survival and recurrence patterns after surgery: MSI, microsatellite stable (MSS) with epithelial-tomesenchymal transition (EMT) signature, MSS with TP53 activation, and MSS without TP53 activation [12]. These classifications have enabled us to improve our understanding of the molecular profiles and heterogeneity of GCs. However, neither of these classifications were designed to optimize patient selection for targeted therapies. Furthermore, comprehensive analyses such as WES and array-based profiling, as performed in the TCGA and ACRG projects, may not be feasible for clinical use due to their higher cost and generation of excessive information about molecular alterations of unknown biological and clinical significance. For clinical genetic testing, which is essential for precision medicine in cancer therapy, panel-based targeted sequencing using NGS technologies is advantageous because of precise target enrichment, enhanced depth of coverage, and reduced cost [14]. Thus, a clinically useful molecular classification based on targeted gene panel sequencing should help to realize precision medicine for GC.
We previously reported the clinical utility of genomic sequencing with a 415-gene panel in colorectal cancer [15]. We expanded the target genes to 435, including 69 genes that are associated with the US Food and Drug Administration (FDA)-approved targeted therapies (actionable genes), and added the evaluation of EBV infection and MSI status using NGS technologies. Here, we generated genomic profiles of 207 Japanese GCs using the 435-gene panel. Further hierarchical clustering based on actionable gene alterations successfully classified GCs into clusters directly associated with promising targeted therapies.

\section{Methods}

\section{Patient inclusion criteria}

A total of 207 patients histologically diagnosed with GC between 2009 and 2015 at Niigata University Medical and Dental Hospital, Niigata Cancer Center Hospital, Gifu University Hospital, Kyushu University Hospital, and Keio University Hospital were enrolled. The clinicopathological data for the individual patients are summarized in Additional file 1: Table S1. The study included 146 males and 61 females, with a median age of 66 years (range, 27-87 years). All patients, except for one patient with liver metastasis, underwent surgical resection with curative intent. Tumors were histologically staged according to the $7^{\text {th }}$ edition of the International Union against Cancer tumor-node-metastasis (TNM) classification system [16]. Collection and use of all specimens in this study were approved by the Institutional Review Boards of Niigata University, Niigata Cancer Center Hospital, Gifu University, Kyushu University, and Keio University. Informed consent was obtained from all subjects.

\section{Sequencing library preparation}

Archival tissue in the form of formalin-fixed, paraffinembedded (FFPE) tumor obtained during routine biopsy $(N=5)$ and/or surgical resection $(N=202)$ was used for analysis. An independent pathologist evaluated tumor content on hematoxylin and eosin-stained slides for each study sample to ensure $>20 \%$ tumor content was present. Where applicable, unstained slides were macrodissected to enrich for tumor content, and DNA was extracted using a BioStic FFPE Tissue DNA Isolation Kit (MO BIO Laboratories, Carlsbad, CA, USA). All sample preparation, genomic sequencing, and analytics were performed in a CLIA/CAP-accredited laboratory (KEW, Cambridge, MA, USA).

\section{Panel-based genomic sequencing}

DNA (50-150 ng) fragment libraries were prepared and enriched for the 435-gene panel with CANCERPLEX (KEW). CANCERPLEX is a clinically validated 435 gene panel enriched for coding regions and selected introns 
of genes with known association in cancer (Additional file 2: Table S2). Sequencing was performed on the Illumina MiSeq and NextSeq platforms (Illumina, San Diego, CA, USA) with average 500× sequencing depth. The CANCERPLEX DNA data sequencing pipeline, GENEPIPER v5.1, implements a series of public and proprietary algorithms designed to analyze DNA sequencing information. The analysis starts with demultiplexing by bcl2fastq v2.17.1.14 from Illumina, in order to obtain one FASTQ file per input sample. The resulting FASTQ files are realigned to the human reference sequence [University of California Santa Cruz (UCSC) hg19/ GRCh37 assembly] using the Burroughs-Wheeler Alignment tool (BWA-MEM) in single-end mode [17, 18]. Duplicated reads resulting from PCR over-amplification or optical duplication are flagged and discarded by the MarkDuplicates module of Picard tools version 2.5 (http://broadinstitute.github.io/picard/).

Somatic and potential germline single nucleotide variants (SNVs) and short insertions and deletions (indels) are called from the cancer tissues using a proprietary combination of somatic and germline variant callers. The models used for this purpose take into account population genetics parameters as well as technical artifacts observed in the panel of normal samples and also statistics from databases such as the Genome Aggregation Database (gnomAD), 1000 Genomes, dbSNP, and COSMIC. All SNV and indel calling, including somatic and pathogenic potentially germline variants, was performed only in genomic regions intended to be captured by the assay (region of interest (ROI)).

Somatic copy number alterations (SCNAs) are called by calculating the number of mapped reads and then performing normalization using the panel of normal samples (49 samples), GC-content, repeat sequence, probe density, and other parameters. Copy number variants are called for exons as well as globally, using a backbone of probes throughout the genome. We segment regions using a Fused-Lasso method and export the results to a VCF file. The threshold for gains was $>2.5$-fold and for loss $<0.75$-fold. Fused genes (structural variants (SVs)) were detected only if at least one end mapped to any of the following genes: AKT3, ALK, BRAF, EGFR, ETV1, ETV4, ETV5, ETV6, FGFR2, FGFR3, MET, NOTCH1, NOTCH2, NRG1, PDGFRA, RAF1, RET, ROS1, TMPRSS2. Variants were filtered or flagged according to technical quality (e.g., coverage, allelic fraction, number of supporting reads), presence in previously characterized normal samples, or presence/absence in the following databases: dbSNP, ExAC, COSMIC, ClinVar, KEW. SNVs and indels in VCF format were annotated using ClinEff 1.0e and hg19 database (UCSC reference with RefSeq identifiers) and the output was adapted according to HGVS recommendations.
The tumor mutation burden (TMB), defined as the rate of peptide-changing SNVs per megabase, was determined for all tumors. To estimate the TMB, after standard filtering had been applied, only SNVs with a mutation allelic fraction of at least $10 \%$ and with high or moderate putative impact were retained. Tumors were tested for the presence of microsatellite instability (MSI) based on an extended loci panel. In addition to the Bethesda panel, a collection of 950 regions consisting of tandem repeats of one, two, or three nucleotides of minimum length of 10 bases. The number of indels within the ROI was calculated and tumors were classified as MSI-high (MSI-H) or microsatellite stable (MSS).

Tumors were also analyzed for the presence of HPV-16, HPV-18, and EBV (HHV-4) viral sequences. The reference genomes used were GI:310698439, GI:9626069, and NC_007605 for HPV-16, HPV-18, and EBV, respectively. The percentage of total number of reads mapped to the viral genomes was calculated and samples were designated as positive based on empirical cutoffs of 0.02, 0.01, and $0.0005 \%$ of reads that were mapped to HPV-16, HPV-18, and EBV genomes, respectively.

The specificity and sensitivity of all variant and biomarker detection was validated as previously described [19]. All non-synonymous genomic changes with low population frequency and allele fraction above 10\% underwent a semi-automated curation and annotation process in GENEKEEPER, a proprietary curation tool and database used to determine pathogenicity and clinical relevance of alterations. We use the catalogue of FDA-approved drugs, the NCCN treatment guidelines, multiple mutation databases, and current scientific literature to determine if the variant protein is a target of a FDA-approved drug, a target of a drug in clinical development, or confers resistance to known treatments. Clinical trials are identified using ClinicalTrial.gov and other tools (e.g., Thompson Reuter Cortellis and Pharma Intelligence Trialtrove). Each variant is classified according the Association of Molecular Pathology (AMP) guidelines for somatic cancer variants [19]. Any variants classified as benign or likely benign were excluded from this study. To align mutations with their protein domains, genomic data were analyzed in Mutation Mapper (http://www.cbioportal.org/) for ERBB2 in Japanese GC.

\section{Genomic profiles of $\mathbf{4 3 5}$ genes in TCGA GCs}

Genomic data and clinicopathological characteristics from a total of 295 tumor samples of GC published in the TCGA database were downloaded from cBioPortal (http://www.cbioportal.org/) for inclusion in this study. To compare the clinicopathological characteristics between Japanese and TCGA GC, two-tailed Fisher's exact test and Mann-Whitney U-test were applied for categorical and continuous variables, respectively. We 
down-sampled whole exome sequencing data to the 435 genes within the CANCERPLEX panel, including mutations $(N=289)$ and putative copy-number alterations from GISTIC $(N=293)$ for tumors that had sequencing data. The Oncoprint analysis tool (http://www.cbiopor tal.org/) was used to plot mutational data among different tumors. We evaluated mutation burden using the mutation data limited to 435 panel genes from TCGA GC samples $(N=289)$. STAD mutation data for the TCGA GCs were downloaded from the Broad GDAC Firehose website (https://gdac.broadinstitute.org/). Similar to the 435-gene panel bioinformatics pipeline, silent mutations that were not protein-altering were removed from the dataset. To compare mutation burden of the 435 gene panel to TCGA WES data on the same samples, the dataset of SNPs was down-sampled to the 435 genes in the panel, and the mutation rate determined in the panel was calculated as mutations/Mb. Correlations between the mutation rate of the 435 genes and of TCGA WES data were evaluated using the Spearman correlation coefficient.

\section{Gene clustering analysis}

Mutation data from non-hypermutated Japanese GCs $(N=175)$ were extracted and clustered by mutated gene patterns using a method described previously [15]. Two different sets of genes-all 435 panel genes and 69 actionable genes-were used for clustering. The number of mutated genes in common related to donors $i$ and $j$ is presented as an element cij of an $N \times \mathrm{N}$ matrix, where $\mathrm{N}$ is the number of non-hypermutated donors. In order to normalize the elements of this $\mathrm{N}$ dimension symmetric matrix into values ranging from 0 to 1 , the original element was replaced by $1 /(c i j+1)$, which indicates the level of similarity between donors $i$ and $j$. Because of this normalization, donors with more mutated-genes in common would more likely come from a relatively close group. Consequently, a matrix with the normalized values between all donors was created. Hierarchical clustering of the matrix was performed for classifying donor groups with different mutated gene patterns by Euclidean distance and Ward's clustering. This clustering was performed using $\mathrm{R}$ (https://www.r-project.org/). Clustering stability was evaluated by $\mathrm{R}$ package clValid for statistical and biological validation of clustering results, and the most stable clusters were determined [20].

\section{Statistical analysis of clinicopathological characteristics}

To estimate associations between co-mutated gene patterns and clinicopathological characteristics, two-tailed Fisher's exact test was applied to categorical variables by comparing the distribution in a cluster group to that of all the donors in the other groups. Mann-Whitney U-test was used for continuous variables. Note that in the case of statistical signature for hypermutated donors, two-tailed Fisher's exact test was conducted against non-hypermutated donors as a reference set. All statistical tests were performed using the $\mathrm{R}$ package statmod (https://cran.r-project.org/web/packages/statmod/index.html), and differences with $P<0.05$ are regarded as statistically significant.

\section{Results}

Overall genomic alterations detected using the 435-gene panel in Japanese GCs

Comprehensive genomic sequencing using the 435-gene panel detected at least one alteration of a cancer-related gene in 194 out of 207 patients (93.7\%). Regarding 69 actionable genes which associated with FDA-approved targeted therapies, at least one alteration was detected in 141 patients (68.1\%). Among 435 genes, mutations (single nucleotide substitutions (SNPs) and/or indels), somatic copy number alterations (SCNAs), and both alterations were found in 173, 31, and 30 genes, respectively. In 207 Japanese GC patients, the most frequently mutated gene was TP53 (53.1\%), followed by ARID1A (15.9\%) and $C D H 1$ (14.0\%). ERBB2 amplification (12.1\%) was the most frequently observed SCNA, followed by CCNE1 (7.2\%) and KRAS (5.8\%) amplification (Table 1).

\section{Molecular classification according to TCGA subtype in Japanese GCs}

The 207 Japanese GCs were classified into four TCGA subtypes based on the genomic profiling data from the 435-gene panel (Fig. 1a). Nine tumors (4\%) positive for EBV-DNA sequence were classified as EBV subtype. MSI-high (MSI-H) status was found in 17 tumors (8\%), and they were classified as MSI subtype. Finally, the remaining 181 tumors were divided into CIN and GS subtypes using SCNA status. In the previous TCGA study, TP53 mutations were frequently observed in the CIN subtype, and tumors with a diffuse type of Lauren classification were enriched in the GS subtype. To match this classification scheme, we defined SCNAhigh tumors as those with four or more SCNA loci (Additional file 3: Figure S1). As a result, 119 SCNAhigh tumors (58\%) were categorized as CIN subtype, and the remaining 62 tumors (30\%) with SCNA-low as GS subtype (Fig. 1b). We compared the distribution of the four subtypes between Japanese and TCGA GCs. Regarding regional differences between enrolled patients, the nationality of $75 \%$ of patients in the TCGA study is European or American (Additional file 3: Figure S2a); on the other hand, all patients included in our study were Japanese. However, the clinicopathological characteristics of each subtype of Japanese GC were roughly comparable to those of the TCGA GCs (Additional file 3: Figure $\mathrm{S} 2 \mathrm{~b}-\mathrm{f}$ ). The proportion of the 
Table 1 Frequent gene alterations in 207 Japanese gastric cancers

\begin{tabular}{|c|c|c|c|c|c|}
\hline Number & $\begin{array}{l}\text { Mutation } \\
\text { gene }\end{array}$ & Frequency & $\begin{array}{l}\text { SCNA } \\
\text { gene }\end{array}$ & Alteration & Frequency \\
\hline 1 & TP53 & $53.1 \%$ & ERBB2 & AMP & $12.1 \%$ \\
\hline 2 & ARIDIA & $15.9 \%$ & CCNE1 & AMP & $6.8 \%$ \\
\hline 3 & $\mathrm{CDH} 1$ & $14.0 \%$ & KRAS & AMP & $5.8 \%$ \\
\hline 4 & $B R C A 2$ & $10.6 \%$ & ZNF217 & AMP & $5.8 \%$ \\
\hline 5 & $A R I D 1 B$ & $10.1 \%$ & $C D K N 2 A$ & DEL & $5.3 \%$ \\
\hline 6 & ATM & $9.7 \%$ & $C D K N 2 B$ & DEL & $5.3 \%$ \\
\hline 7 & PIK3CA & $8.7 \%$ & GATA4 & AMP & $4.3 \%$ \\
\hline 8 & $A P C$ & $8.2 \%$ & MYC & AMP & $2.4 \%$ \\
\hline 9 & $A C V R 2 A$ & $7.2 \%$ & CCND3 & AMP & $1.9 \%$ \\
\hline 10 & CHD2 & $6.3 \%$ & $C D 274$ & AMP & $1.9 \%$ \\
\hline 11 & KMT2D & $6.3 \%$ & CDK6 & AMP & $1.9 \%$ \\
\hline 12 & RNF43 & $5.8 \%$ & $E G F R$ & AMP & $1.9 \%$ \\
\hline 13 & EPHA2 & $5.8 \%$ & FGFR2 & AMP & $1.9 \%$ \\
\hline 14 & TGFBR2 & $5.3 \%$ & $J A K 2$ & AMP & $1.9 \%$ \\
\hline 15 & FLCN & $4.3 \%$ & GNAS & AMP & $1.9 \%$ \\
\hline 16 & PALB2 & $4.3 \%$ & CCND1 & AMP & $1.4 \%$ \\
\hline 17 & PTPRT & $4.3 \%$ & MET & AMP & $1.4 \%$ \\
\hline 18 & RAD50 & $4.3 \%$ & HSP9OAB1 & AMP & $1.4 \%$ \\
\hline 19 & $B R C A 1$ & $3.9 \%$ & SMAD4 & DEL & $1.4 \%$ \\
\hline 20 & STK11 & $3.9 \%$ & TEK & DEL & $1.4 \%$ \\
\hline
\end{tabular}

AMP amplification, DEL deletion, SCNA somatic copy number alteration Bold gene symbols indicate genes from the 69 actionable genes for FDA-approved targeted therapies

MSI subtype was lower, and that of the GS subtype was higher, in Japanese GCs than in TCGA GCs $(P<0.01$; Fig. 1c). Because genomic profiling of actionable genes may lead to strategies for optimal targeted therapies in $\mathrm{GC}$, we examined actionable gene alterations that were observed at a frequency of $5 \%$ or more in Japanese GCs. Interestingly, these alterations were not specific to a TCGA subtype (Fig. 1d).

\section{Hypermutated tumors in Japanese GCs}

Recently, hypermutated tumors have been considered to have a higher response to immunotherapy because of the development of neo-antigens [21, 22]. In the previous TCGA study of GC, hypermutated tumors were defined as MSI-H tumors [10]. The mutation rate of MSI-H tumors (31.5 counts/Mb, range 18.5-66.9) was significantly higher than that of MSS tumors (11.2 counts/Mb, range 3.1-27.7) in Japanese GCs $(P<0.01)$. We defined hypermutated tumors as those with a mutation rate of more than 18.5 counts $/ \mathrm{Mb}$, which was the lowest value in MSI-H tumors (Fig. 2). To validate the cutoff value of mutation rate, WES data of TCGA GCs was down-sampled to the 435 genes, and mutation rate calculated. A high correlation of mutation rate between WES and the 435-gene panel was demonstrated (correlation coefficient of 0.966). The lowest mutation rate of MSI-H tumors in TCGA GCs was 16.4 counts $/ \mathrm{Mb}$, and was nearly equivalent to that in Japanese GCs. As a result, we identified 32 tumors (15.5\%) as hypermutated tumors, which not only included the MSI subtype but also some tumors from the EBV, CIN, and GS subtypes (Fig. 1a).

\section{Clustering based on gene alterations for targeted therapy in Japanese GCs}

Actionable gene alterations and hypermutated phenotypes were widely observed throughout all TCGA subtypes in our analysis (Fig. 1a, d). Novel classifications, directly associated with these alterations, may better pinpoint candidates for targeted therapies than TCGA subtypes. Thus, our 207 tumors were classified using the hypermutated phenotype and alteration profile of the 69 actionable genes. Hypermutated tumors $(N=32)$ were identified, and the remaining non-hypermutated tumors $(N=175)$ were subdivided into six clusters by hierarchical clustering (Fig. 3). Firstly, tumors with ERBB2 alterations were classified as cluster $1(N=25)$. Remaining tumors without $E R B B 2$ alterations were classified into two large clusters according to the enrichment of actionable gene alterations. The cluster with enrichment of the major alterations were subsequently divided into four clusters (clusters $2-5$ ) based on intrinsic gene alterations in $C D K N 2 A / B(N=10), \operatorname{KRAS}(N=10), \operatorname{BRCA2}(N=9)$, or $\operatorname{ATM}(N=12)$. Cluster $6(N=109)$ included tumors with minor alterations and those with no alterations in actionable genes. Using this clustering scheme, FDAapproved targeted therapies could be associated with a majority of tumors, including hypermutated tumors, those in clusters $1-5$ and a part of cluster 6 .

In addition, we performed hierarchical clustering based on the 435-gene alterations to assess the associations between overall genomic profile and clinicopathological features. Non-hypermutated tumors were classified into nine clusters (Additional file 3: Figure S3a). We identified the clusters which have specific clinicopathological features (Additional file 3: Figure S3b). Tumors in cluster $9(N=16)$, which are characterized by concomitant alterations of TP53 and ERBB2, significantly associated with high counts of SCNA $(P<0.01)$ and the intestinal type of Lauren classification $(P<0.05)$ compared with tumors in all other clusters. On the other hand, tumors in cluster 4 with primary mutated gene $\mathrm{CDH} 1$ significantly associated with young age $(P<0.05)$, low counts of SCNA $(P<0.01)$, female gender $(P<0.01)$, and diffuse type of Lauren classification $(P<0.01)$. These findings suggest that genomic profiling generated by the 435-gene panel reflects the clinicopathological features in Japanese GCs. 


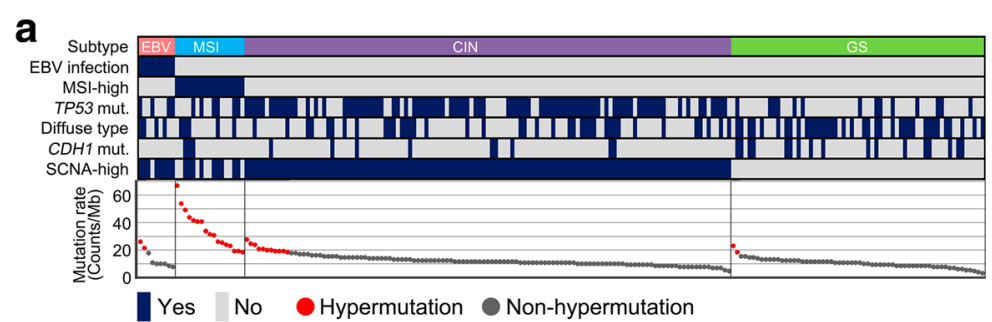

b

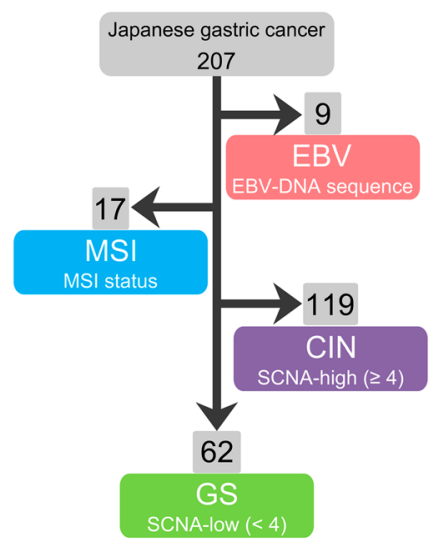

C Japanese gastric cancer

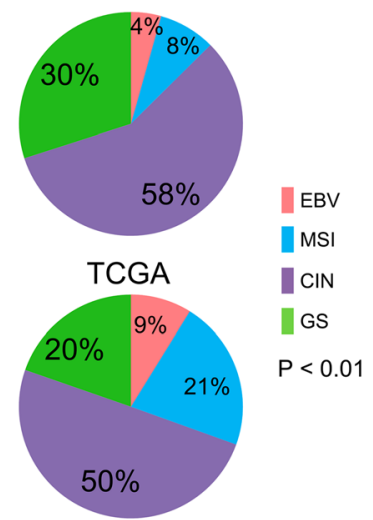

d

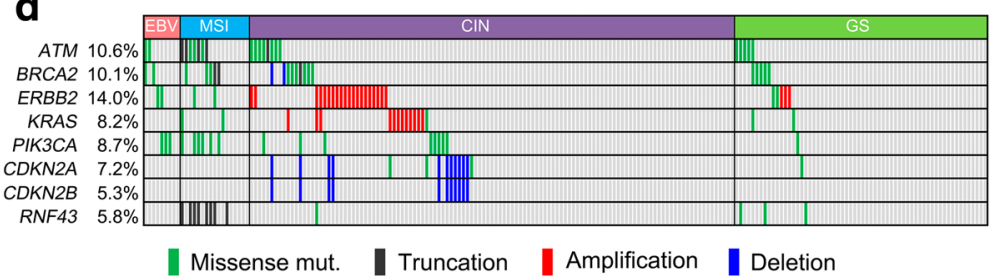

Fig. 1 The Cancer Genome Atlas molecular subtypes in 207 Japanese gastric cancers (GCs). a The 207 Japanese GCs are classified into four molecular subtypes; Epstein-Barr virus-positive (EBV, red), tumor with microsatellite instability (MSI, blue), tumors with chromosomal instability (CIN, purple), and genomically stable tumors (GS, green). Color tiles indicate pathological and molecular characteristics of of GC. Tumors are ordered by mutation rate and the red dots indicate hypermutated tumors with a mutation rate of more than 18.5 counts/Mb, which was the lowest value in the MSI subtype. b Classification of 207 Japanese GCs into TCGA molecular subtypes. The cutoff value of somatic copy number alterations (SCNAs) is defined as four loci according to the status of TP53 mutation and histological type (Additional file 3: Figure S1). c Distribution of molecular subtypes in 207 Japanese (upper) and TCGA (lower) GCs. d Landscape of frequently observed actionable gene alterations (5\% or more) in 207 Japanese GCs classified by TCGA subtype. Alteration color indicates the class of gene alterations

\section{Details of gene alterations and a remarkable case in the ERBB2 cluster (cluster 1)}

Anti-HER2 therapy with trastuzumab is recommended as a first line therapy for HER2-overexpressing GC [5]. Tumors harboring ERBB2 alterations were primarily assigned into cluster 1 in our study. $E R B B 2$ amplification, mutation, and both alterations were detected in 22, two, and one tumor, respectively. Details of ERBB2 mutations in the Japanese GCs are shown in Fig. 4a. All the mutations detected in cluster 1 were S310F, which is well-known to activate HER2 signaling [23, 24]. Tumors with the S310F mutation have been reported to have good response to anti-HER2 therapy [25].

Within cluster 1 , one patient demonstrated a remarkable response to anti-HER2 therapy (Fig. 4b-d). In brief, this patient was a 55-year old woman with unresectable GC because of extensive abdominal and paraaortic lymph node metastases. HER2 overexpression was confirmed by immunohistochemistry in the biopsy specimen, and trastuzumab combined with chemotherapy was administered. After seven courses of treatment, complete response according to RECIST ver. 1.1 was observed in abdominal and paraaortic lymph node metastases. The primary tumor of the stomach also decreased in size, although histological examination of the endoscopic biopsy specimen revealed residual tumor. A PET-CT scan showed abnormal uptake only in the primary tumor of the stomach. Therefore, we performed distal gastrectomy with regional and paraaortic lymphadenectomy, and achieved curative 

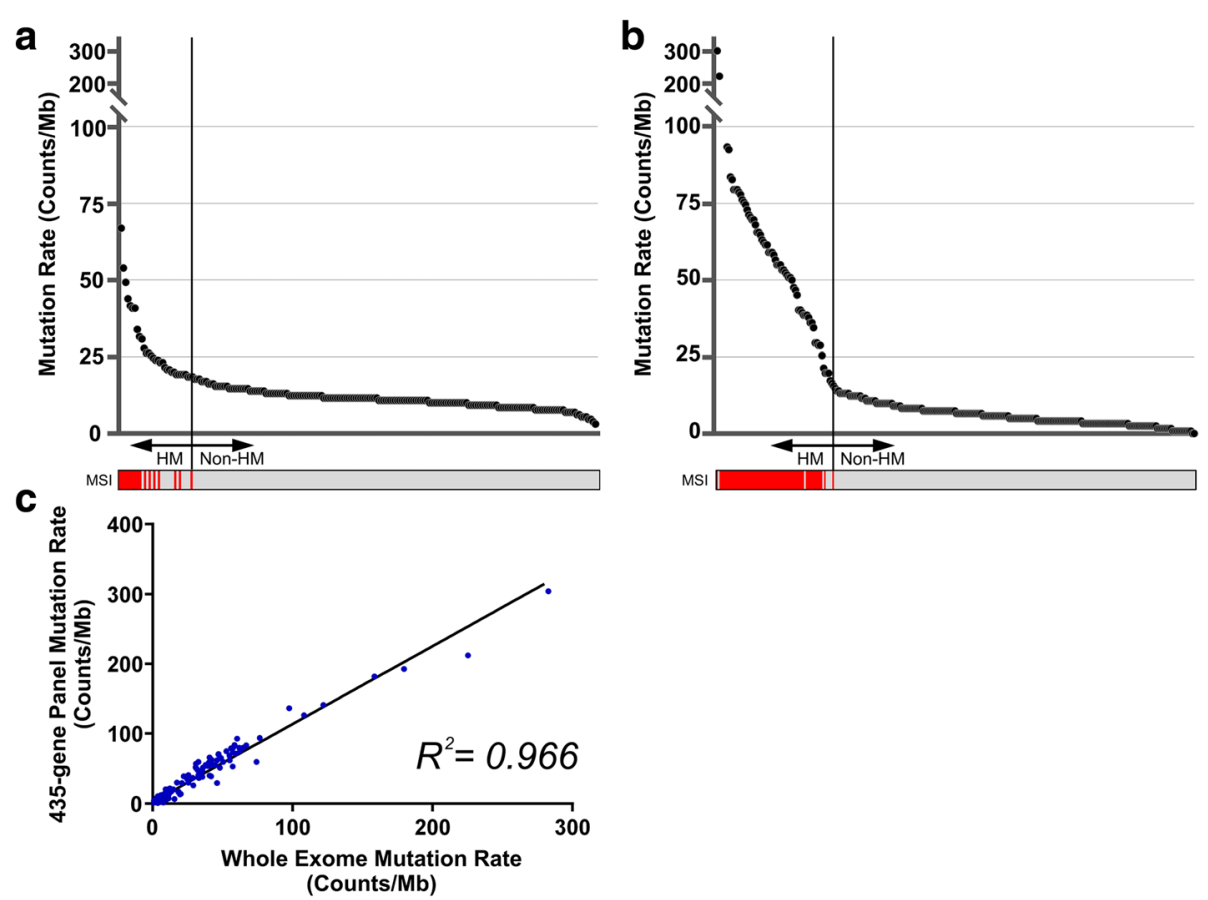

Fig. 2 Mutation rates in Japanese and The Cancer Genome Atlas gastric cancers. a Mutation rate from Japanese GCs was determined by the number of non-synonymous SNVs in the 435-gene panel. Red, MSI-H; gray, MSS; HM, hypermutation. b WES data from TCGA GCs was down-sampled to the content of the 435-gene panel. Red, MSI-H; gray, MSS; HM, hypermutation. c Correlation between mutation rates determined using the 435-gene panel and WES data of TCGA GCS

resection. Histological examination revealed no viable tumor cells in the abdominal and paraaortic lymph nodes. Genomic alterations in the biopsy specimen obtained before treatment were retrospectively examined using the 435-gene panel. Consistent with the immunohistochemistry, ERBB2 amplification was detected. Other alterations detected were ZNF217 amplification and TP53 mutation. Concomitant alterations in the ERBB2 downstream pathway, which confer resistance to trastuzumab therapy, were not observed in the tumor of this patient.

\section{Discussion}

GC is a highly heterogeneous disease with various histological phenotypes and molecular diversity. Intertumoral heterogeneity represents a significant hurdle to identifying optimized targeted therapies in GC. Stratification of patients based upon the distinctive genomic alterations in their tumors is needed to realize precision medicine in GC. To the best of our knowledge, the current study is the first to demonstrate the actionable gene-based molecular classification associated with FDA-approved targeted therapies using panel-based targeted sequencing. We generated comprehensive genomic profiling data for 207 Japanese GCs using a 435-gene panel including the evaluation of EBV infection and MSI status based on NGS technologies. We identified five clusters by hierarchical clustering according to alterations in 69 actionable genes in addition to hypermutated status, which are associated with FDA-approved targeted therapies. Our study highlighted the potential of panel-based targeted sequencing to provide reliable information for realizing precision medicine in GC.

Large-scale genome projects have attempted to identify distinct molecular subtypes to help define the heterogeneity of GCs; however, a clinically useful classification that is relevant to targeted therapies is yet to be found for GC. TCGA molecular subtypes are a reasonable classification which reflects the tumor biology and associates with clinicopathologic features of GC [10]. ACRG molecular subtypes, established using gene expression and genome-wide copy number microarray data, were associated with survival outcome and recurrence patterns in patients with GC after surgery [12]. However, it remains unclear whether these molecular subtypes can provide optimum targeted therapies in GC. In this study, 207 Japanese GCs were classified into TCGA molecular subtypes, and we showed that alterations in actionable genes are not specific to TCGA molecular subtype, but rather are widely distributed throughout the subtypes. Therefore, TCGA molecular subtype may not be directly useful for clinical applications to optimize targeted therapies. Novel classifications 


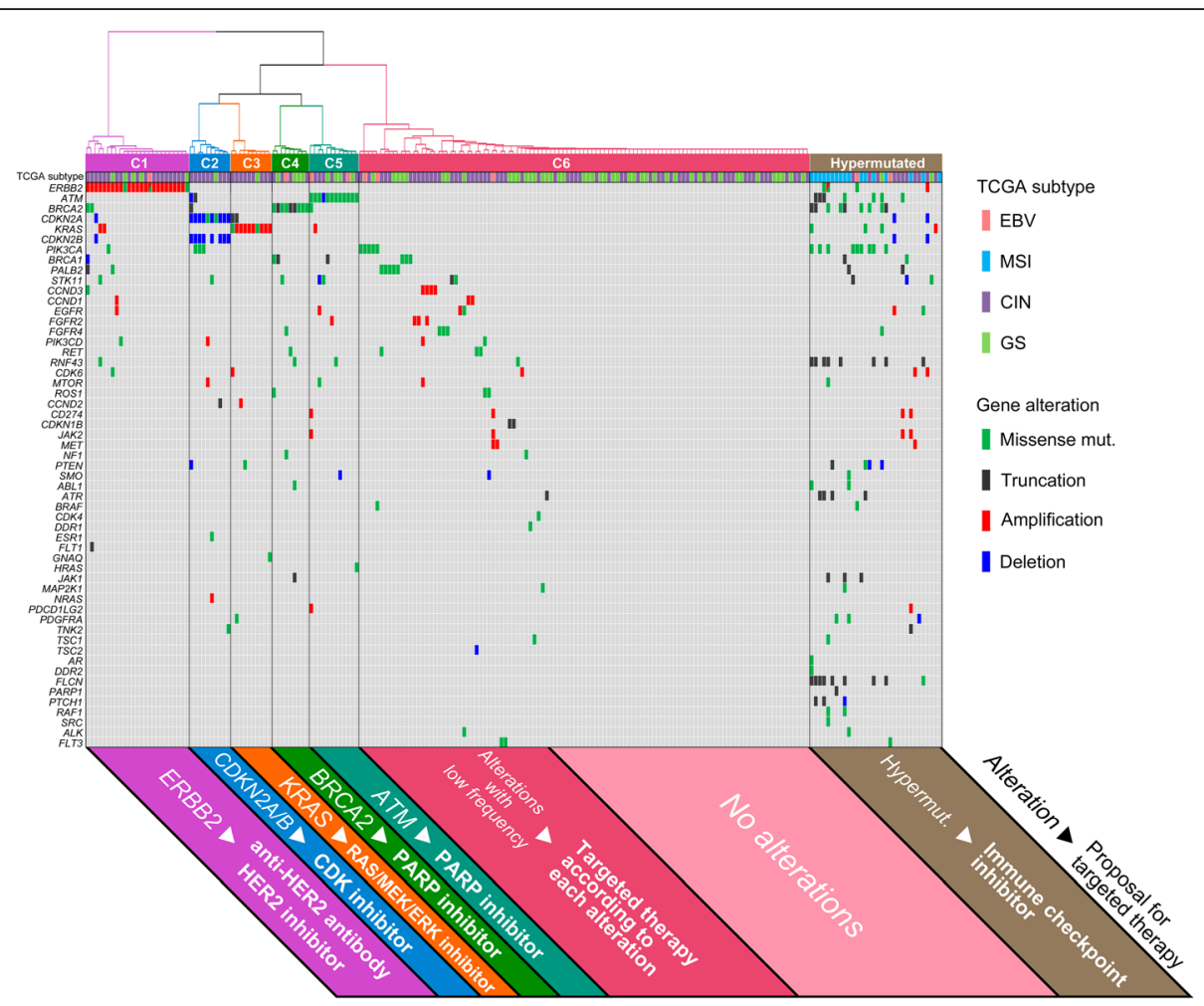

Fig. 3 Clustering based on 69 actionable gene alterations. According to co-alteration patterns of the 69 actionable gene subset, 175 non-hypermutated tumors are divided into six clusters, and the gene alteration spectrum and TCGA molecular subtype of each tumor is demonstrated as color tiles. Alteration in color indicates the class of gene alteration. The 32 hypermutated tumors are shown on the right (brown). The specific gene alteration and proposal for targeted therapy are presented under each cluster. Fourteen genes which have no alterations in 207 tumors are not included in this figure

which more precisely select candidates for targeted therapies are necessary to realize precision medicine in GC.

We successfully classified 207 tumors into clusters directly associated with FDA-approved targeted therapies according to the alterations of 69 actionable genes and hypermutated phenotype. We identified the clusters with enrichment of $C D K N 2 A / B, K R A S, A T M$, or BRCA2 alterations, in addition to ERBB2 ones. ATM and BRCA1/ 2 play an essential role in double-strand DNA break repair pathway, and the deficiency of this pathway caused by the alterations of these genes were associated with high sensitivity to the PARP inhibitors in solid tumors, including GC $[26,27]$. A phase II clinical trial revealed the efficacy of olaparib as a second line therapy in recurrent or metastatic GC with negative expression of ATM protein by immunohistochemistry [28]. However, phase III clinical trial did not show the significant overall survival benefit of olaparib in this population (NCT01924533) [29]. Further clinical studies of PARP inhibitors for BRCA1/2 or ATM mutated GCs are necessary to establish the appropriate biomarker and to reveal the benefit of this therapy. KRAS mutation and amplification lead to an activation of the RAF-MEK-ERK pathway, which is critical to pathogenesis and progression in many cancers [30]. KRAS itself is difficult to directly inhibit; therefore, inhibition of the downstream RAFMEK-ERK pathway is considered a promising treatment for KRAS-mutated tumors [31]. A clinical benefit of the FDA-approved MEK inhibitor trametinib has been partially demonstrated in cancers with a KRAS mutation $[32,33]$. CDKN2A/B protein products restrict cell-cycle progression by inhibiting CDK4/6 kinase activity, and aberrations in these genes lead to cancer progression by dysregulation of the cell cycle [34]. The association between $C D K N 2 A / B$ deletion and the sensitivity to palbociclib, an FDA-approved CDK4/6 inhibitor, has been demonstrated in many cancers [35-38]. Therefore, it is worth investigating the clinical utility of the abovementioned targeted therapies for GCs with corresponding gene alterations to establish precision medicine in GC.

Our hierarchical clustering based on actionable gene alterations identified the cluster with $E R B B 2$ alterations as one of the major clusters. Patients with tumors harboring ERBB2 amplification $(N=22)$ are candidates for trastuzumab, an anti-HER2 monoclonal antibody, as a standard first line therapy for unresectable metastatic GC. Excitingly, a remarkable response to trastuzumab therapy was observed in one of the ERBB2-amplified 

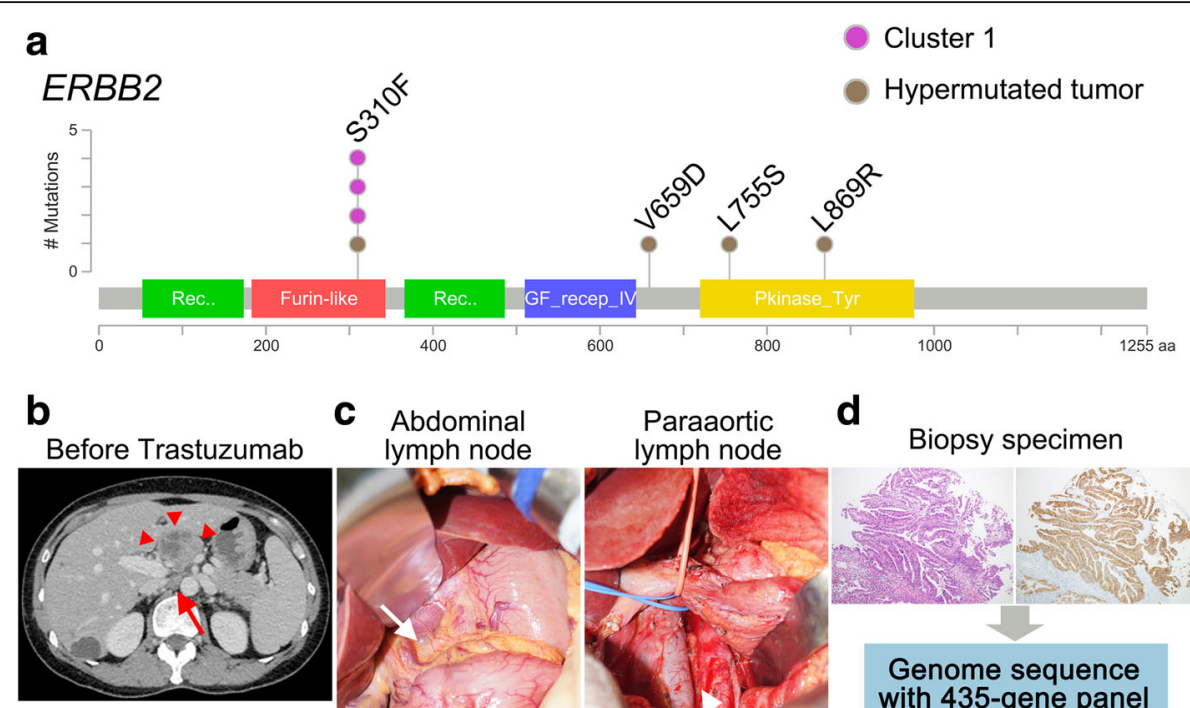

d
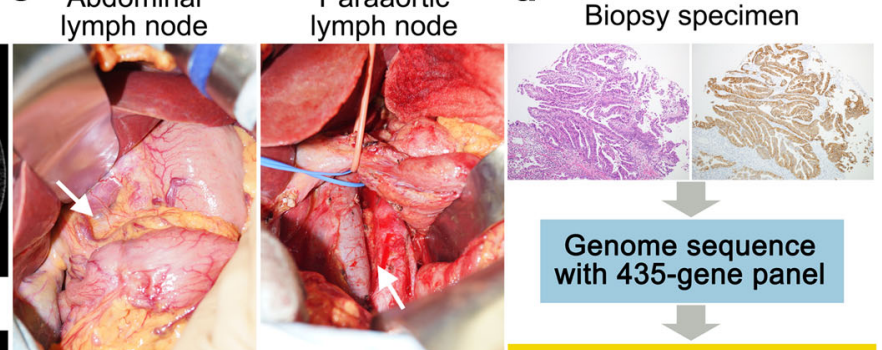

Genome sequence with 435-gene panel
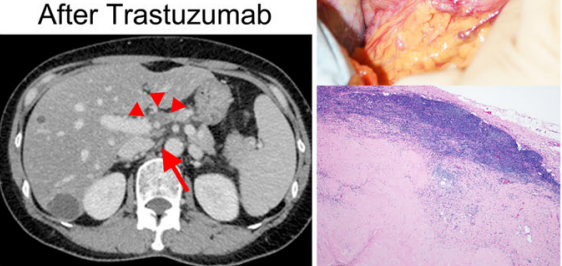

\section{ERBB2 amplification \\ ZNF217 amplification \\ TP53 mutation \\ No alterations in ERBB2 downstream pathway}

Fig. 4 Details of ERBB2 alterations and a case of remarkable response to anti-HER2 therapy in the ERBB2 cluster. a Mutations of ERBB2 identified in 207 Japanese GCs are aligned to the protein domain. Patient samples are further plotted by mutation status. b Abdominal enhanced CT before trastuzumab therapy (upper panel) demonstrated extensive metastases in abdominal (arrow head) and paraaortic (arrow) lymph nodes. All the metastatic lymph nodes were remarkably reduced in size after trastuzumab therapy (lower panel). c Abdominal lymph nodes were reduced in size, and paraaortic lymphadenectomy was performed (upper panel). Histological examination revealed no viable tumor cells in the abdominal and paraaortic lymph nodes (lower panel, hematoxylin and eosin, original magnification $\times 40$ ). d Genomic sequencing in biopsy specimen obtained before treatment (left panel, hematoxylin and eosin; right panel, anti-HER2 antibody) identified ERBB2 and ZNF217 amplification and TP53 mutation. However, concomitant alterations in the ERBB2 downstream pathway were not observed in the tumor of this patient

tumors categorized into this cluster, and genomic sequencing identified no concomitant alterations in the ERBB2 downstream pathway. Alterations in the PI3K/ mTOR and MAPK pathways and amplification of other receptor tyrosine kinases are proposed mechanisms of trastuzumab resistance [39-41]. Genomic profiling using the 435-gene panel enabled us to simultaneously examine the gene alterations of these pathways in single testing, and has the potential to provide reliable information to predict treatment response and to overcome resistance for targeted therapies [42, 43].

Recent advances on the genetics of GC are pointing toward an enrichment of the mutations involved in double-strand DNA break repair via homologous recombination (HR). Alexandrov et al. [44] demonstrated that $7-12 \%$ of GCs have deficiencies in this pathway using a large-scale mutational signatures analysis. Sahasrabudhe et al. [45] identified 11 of $362 \mathrm{GC}$ cases with germline mutations in PALB2, BRCA1, or RAD51C, which regulate HR. In this study, we identified the high frequency of mutations in HR genes, including BRCA1/2, ATM,
PALB2, and RAD50 (Table 1). This results indicates that defective HR is a crucial genetic aberration in Japanese GCs, similar to recent studies. Ovarian and breast cancers with defective HR have high sensitivity to platinum agents as well as PARP inhibitors [46, 47]. Platinumbased chemotherapy is a mainstay of current treatment for advanced GC $[2,4]$. Therefore, the assessment of defective HR using panel-based targeted sequencing might allow optimization of patient selection for platinumbased chemotherapy in GC.

Previous comprehensive studies characterized diffuse type GCs as tumors with a low frequency of mutation and SCNA, which were classified as GS subtype in the TCGA study [10] or MSS/EMT subtype in the ACRG study [12]. Promising therapeutic targets are hard to identify in these subtypes because of the genomic stability. Indeed, in our hierarchical clustering analysis, diffuse type and GS tumors were significantly enriched in 63 tumors with no actionable alterations which associated with FDA-approved targeted therapies: 52.4 and 46.0\%, respectively (Additional file 3: Figure S4). Several studies 
using WES or WGS focusing on diffuse type GCs were conducted to understand the molecular background and to explore therapeutic targets $[9,48]$. However, promising targeted therapies are not currently developed in diffuse type GCs. Further challenges exist in developing effective targeted therapies for these tumors with genomic stability.

Except for trastuzumab, targeted therapies proposed by our actionable gene-based classification are not currently approved for GC. Future studies are needed to determine the effectiveness of actionable gene-based classifications to improve patient outcomes. Clinical trials for targeted therapies, coupled with genomic profiling for optimum patient selection, are required to demonstrate clinical utility, including treatment outcome and cost-effectiveness. Nonetheless, we believe that our genomic profiling data and classification are informative for future studies to explore the rationale for precision medicine in GC because our study represents the most extensive large-scale targeted sequencing project in Japan where a high incidence of GC is observed.

\section{Conclusions}

We generated genomic profiling data for 207 Japanese GCs using the 435-gene panel with NGS technology. Our actionable gene-based classification creates a framework for further studies aimed at realizing precision medicine in GC.

\section{Additional files}

Additional file 1: Table S1. Clinicopathological characteristics of 207 Japanese gastric cancers. (XLSX $38 \mathrm{~kb}$ )

Additional file 2: Table S2. Detail of the 435-gene panel. (XLSX $16 \mathrm{~kb}$ ) Additional file 3: Figures S1-S4. Figure S1. Definition of somatic copy number alteration (SCNA) status. Figure S2. Clinicopathological characteristics of TCGA molecular subtype in Japanese and TCGA gastric cancers (GCs). Figure S3. Cluster of 435-gene co-mutation patterns. Figure S4. Distribution of Lauren classification and TCGA molecular subtypes by presence or absence of actionable gene alterations. (PDF $725 \mathrm{~kb}$ )

\section{Abbreviations \\ ACRG: The Asian Cancer Research Group; CIN: Chromosomal instability; EBV: Epstein-Barr virus; EMT: Epithelial-to-mesenchymal transition; FDA: US Food and Drug Administration; FFPE: Formalin-fixed, paraffin embedded; GC: Gastric cancer; GS: Genomically stable; HR: Homologous recombination; Indel: Insertion/deletion; MSI: Microsatellite instability; MSS: Microsatellite stable; NGS: Next-generation sequencing; ROI: Region of interest; SCNA: Somatic copy number alteration; SNP: Single nucleotide substitution; SNV: single nucleotide variant; TCGA: The Cancer Genome Atlas; TMB: Tumor mutation burden; TNM: Tumor-node-metastasis; WES: Whole-exome sequencing.}

\section{Acknowledgements}

We thank our colleagues Masaki Aizawa, Atsushi Matsuki, Takashi Kawasaki, and Keiichi Homma. We greatly appreciate Jennifer E. Ring and Alexei Protopopov for genomic sequencing.

\section{Funding}

This project was supported by funding from Denka Co., Ltd. H. Ichikawa is supported, in part, by the Japan Society for the Promotion of Science (JSPS) KAKENHI grant number JP16K10491. M. Nagahashi is supported by the JSPS KAKENHI grant numbers JP15H05676 and JP15K15471, the Uehara Memorial Foundation, Nakayama Cancer Research Institute, Takeda Science

Foundation, and Tsukada Medical Foundation. T. Wakai is supported by the JSPS KAKENHI grant numbers JP15H04927 and JP16K15610. K. Yoshida is supported by the JSPS KAKENHI grant number JP17H04280. S. Okuda is supported by the JSPS KAKENHI grant number JP26700029. K. Takabe is supported by NIH/NCl grant R01CA160688 and Susan G. Komen Investigator Initiated Research Grant IIR12222224. S. Lyle is supported by a grant from the Massachusetts Life Sciences Center.

\section{Availability of data and materials}

The datasets generated and/or analyzed during the current study are not publicly available due to data and privacy protection considerations but may be available on justified request. Investigators wishing to download controlledaccess data are expected to abide by the Genomic Data User Code of Conduct through their agreement to the Data Use Certification. The Data Use Certification co-signed by the investigators requesting the data and their Institutional Signing Official, specifies the conditions for the secondary research use of controlledaccess data, including: Using the data only for the approved research; Protecting data confidentiality; Following, as appropriate, all applicable national, tribal, and state laws and regulations, as well as relevant institutional policies and procedures for handling genomic data; Not attempting to identify individual participants from whom the data were obtained; Not selling any of the data obtained from data repositories; Not sharing any of the data obtained from controlled-access data repositories with individuals other than those listed in the data access request; Agreeing to report any violation of the GDS Policy to the appropriate DAC(s) as soon as it is discovered; Reporting research progress using controlled-access datasets through annual access renewal requests or project close-out reports; Acknowledging in all oral or written presentations, disclosures, or publications the contributing investigator(s) who conducted the original study, the funding organization(s) that supported the work, and the specific dataset(s) and applicable accession number(s). The raw data for the TCGA GC samples (STAD) are available at the Broad GDAC Firehose website (https://gdac.broadinstitute.org/).

\section{Authors' contributions}

$\mathrm{HI}, \mathrm{YK}, \mathrm{KY}, \mathrm{EO}$, and TW were project leaders. HI, MN, DV, SL, SO, KT, and TW wrote the manuscript. TH, TI, YS, HK, TK, JS, HY, SN, NS, YH, YK, TT, KY, RN, and EO analyzed clinicopathological data. HI, DV, SL, YL, and SO examined genomic alterations and pathways. DV, YL, and SO performed bioinformatics analysis. KA supervised the statistical analysis. All authors read and approved the final manuscript.

\section{Ethics approval and consent to participate}

This study was conducted in accordance with the provisions of the Declaration of Helsinki. Collection and use of all specimens in this study were approved by the Institutional Review Boards of Niigata University Graduate School of Medical and Dental Sciences (\#771), Niigata Cancer Center Hospital (\#641), Gifu University (\#27-326), Kyushu University (\#672-00), and Keio University (\#20150469). Informed consent was obtained from all participants.

\section{Consent for publication}

Informed consent for the presentation of case reports was obtained from patients.

\section{Competing interests}

SL and DV are employees of and have been granted stock options by KEW Inc. The remaining authors declare that they have no competing interests.

\section{Publisher's Note}

Springer Nature remains neutral with regard to jurisdictional claims in published maps and institutional affiliations.

\section{Author details}

'Division of Digestive and General Surgery, Niigata University Graduate School of Medical and Dental Sciences, 1-757 Asahimachi-dori, Chuo-ku, Niigata City, Niigata 951-8510, Japan. ${ }^{2}$ Department of Gastroenterological 
Surgery, Niigata Cancer Center Hospital, 2-15-3 Kawagishi-cho, Chuo-ku, Niigata City, Niigata 951-8566, Japan. ${ }^{3}$ Department of Breast Oncology, Niigata Cancer Center Hospital, 2-15-3 Kawagishi-cho, Chuo-ku, Niigata City, Niigata 951-8566, Japan. ${ }^{4}$ Department of Surgery, Keio University School of Medicine, 35 Shinano-machi, Shinjyuku-ku, Tokyo 160-8582, Japan. ${ }^{5}$ Department of Surgical Oncology, Gifu University Graduate School of Medicine, 1-1 Yanagido, Gifu 501-1194, Japan. ${ }^{6}$ Department of Surgery and Science, Graduate School of Medical Sciences, Kyushu University, 3-1-1 Maidashi, Higashi-ku, Fukuoka 812-8582, Japan. Division of Genetics, Department of Medicine, Brigham and Women's Hospital and Harvard Medical School, Boston, Massachusetts 02115, USA. ${ }^{8}$ Broad Institute of Harvard and MIT, Cambridge, Massachusetts 02142, USA. ${ }^{9}$ Molecular, Cell \& Cancer Biology, University of Massachusetts Medical School, 55 Lake Avenue North, Worcester, Massachusetts 01655, USA. ${ }^{10}$ Breast Surgery, Roswell Park Cancer Institute, Elm \& Carlton Streets, Buffalo, New York 14263, USA. ${ }^{11}$ Department of Surgery, University at Buffalo the State University of New York, 100 High Street, Buffalo, New York 14203, USA. ${ }^{12}$ Division of Bioinformatics, Niigata University Graduate School of Medical and Dental Sciences, 1-757 Asahimachi-dori, Chuo-ku, Niigata City, Niigata 951-8510, Japan. ${ }^{13}$ Department of Medical Informatics, Niigata University Medical and Dental Hospital, 1-757 Asahimachi-dori, Chuo-ku, Niigata City, Niigata 951-8510, Japan.

Received: 17 July 2017 Accepted: 17 October 2017 Published online: 31 October 2017

\section{References}

1. Ferlay J, Soerjomataram I, Dikshit R, Eser S, Mathers C, Rebelo M, et al. Cancer incidence and mortality worldwide: sources, methods and major patterns in GLOBOCAN 2012. Int J Cancer. 2015;136:E359-86.

2. Koizumi W, Narahara H, Hara T, Takagane A, Akiya T, Takagi M, et al. S-1 plus cisplatin versus S-1 alone for first-line treatment of advanced gastric cancer (SPIRITS trial): a phase III trial. Lancet Oncol. 2008;9:215-21.

3. Fujitani K, Yang HK, Mizusawa J, Kim YW, Terashima M, Han SU, et al. Gastrectomy plus chemotherapy versus chemotherapy alone for advanced gastric cancer with a single non-curable factor (REGATTA): a phase 3, randomised controlled trial. Lancet Oncol. 2016:17:309-18.

4. Harada K, Mizrak Kaya D, Shimodaira Y, Ajani JA. Global chemotherapy development for gastric cancer. Gastric Cancer. 2017;20:92-101.

5. Bang YJ, Van Cutsem E, Feyereislova A, Chung HC, Shen L, Sawaki A, et al. Trastuzumab in combination with chemotherapy versus chemotherapy alone for treatment of HER2-positive advanced gastric or gastrooesophageal junction cancer (ToGA): a phase 3, open-label, randomised controlled trial. Lancet. 2010;376:687-97.

6. Fuchs CS, Tomasek J, Yong CJ, Dumitru F, Passalacqua R, Goswami C, et al. Ramucirumab monotherapy for previously treated advanced gastric or gastrooesophageal junction adenocarcinoma (REGARD): an international, randomised, multicentre, placebo-controlled, phase 3 trial. Lancet. 2014;383:31-9.

7. Wilke H, Muro K, Van Cutsem E, Oh SC, Bodoky G, Shimada Y, et al. Ramucirumab plus paclitaxel versus placebo plus paclitaxel in patients with previously treated advanced gastric or gastro-oesophageal junction adenocarcinoma (RAINBOW): a double-blind, randomised phase 3 trial. Lancet Oncol. 2014;15:1224-35.

8. Wang K, Yuen ST, Xu J, Lee SP, Yan HHN, Shi ST, et al. Whole-genome sequencing and comprehensive molecular profiling identify new driver mutations in gastric cancer. Nat Genet. 2014;46:573-82

9. Kakiuchi M, Nishizawa T, Ueda H, Gotoh K, Tanaka A, Hayashi A, et al. Recurrent gain-of-function mutations of RHOA in diffuse-type gastric carcinoma. Nat Genet. 2014:46:583-7.

10. The Cancer Genome Atlas Research Network. Comprehensive molecular characterization of gastric adenocarcinoma. Nature. 2014;513:202-9.

11. Chen K, Yang D, Li X, Sun B, Song F, Cao W, et al. Mutational landscape of gastric adenocarcinoma in Chinese: implications for prognosis and therapy. Proc Natl Acad Sci U S A. 2015;112:1107-12.

12. Cristescu R, Lee J, Nebozhyn M, Kim KM, Ting JC, Wong SS, et al. Molecular analysis of gastric cancer identifies subtypes associated with distinct clinical outcomes. Nat Med. 2015;21:449-56.

13. Kuboki Y, Yamashita S, Niwa T, Ushijima T, Nagatsuma A, Kuwata T, et al. Comprehensive analyses using next-generation sequencing and immunohistochemistry enable precise treatment in advanced gastric cancer. Ann Oncol. 2016;27:127-33.
14. Horak P, Fröhling S, Glimm H. Integrating next-generation sequencing into clinical oncology: strategies, promises and pitfalls. ESMO Open. 2016;1:e000094

15. Nagahashi M, Wakai T, Shimada Y, Ichikawa H, Kameyama H, Kobayashi T, et al. Genomic landscape of colorectal cancer in Japan: clinical implications of comprehensive genomic sequencing for precision medicine. Genome Med. 2016:8:136.

16. Sobin LH, Gospodarowicz MK, Wittekind C. TNM classification of malignant tumours (Uicc International Union Against Cancer). 7th ed. Oxford: WileyBlackwell; 2010

17. Li H, Durbin R. Fast and accurate short read alignment with BurrowsWheeler transform. Bioinformatics. 2009;25:1754-60.

18. Li H. Exploring single-sample SNP and INDEL calling with whole-genome de novo assembly. Bioinformatics. 2012;28:1838-44.

19. Eifert C, Pantazi A, Sun $R, X u$ J, Cingolani P, Heyer J, et al. Clinical application of a cancer genomic profiling assay to guide precision medicine decisions. Per Med. 2017;14:309-25.

20. Brock G, Pihur V, Datta SS, Datta SS. cIValid: an R package for cluster validation. J Stat Softw. 2008;25:1-22.

21. Rizvi NA, Hellmann MD, Snyder A, Kvistborg P, Makarov V, Havel JJ, et al. Mutational landscape determines sensitivity to PD-1 blockade in non-small cell lung cancer. Science. 2015;348:124-8.

22. Le DT, Uram JN, Wang H, Bartlett BR, Kemberling H, Eyring AD, et al. PD-1 blockade in tumors with mismatch-repair deficiency. N Engl J Med. 2015; 372:2509-20.

23. Greulich H, Kaplan B, Mertins P, Chen T, Tanaka KE, Yun C, et al. Functional analysis of receptor tyrosine kinase mutations in lung cancer identifies oncogenic extracellular domain mutations of ERBB2. Proc Natl Acad Sci U S A. 2012:109:14476-81.

24. Bose R, Kavuri SM, Searleman AC, Shen W, Shen D, Koboldt DC, et al. Activating HER2 mutations in HER2 gene amplification negative breast cancer. Cancer Discov. 2013;3:224-37.

25. Chumsri S, Weidler J, Ali S, Balasubramanian S, Wallweber G, De Fazio-Eli L, et al. Prolonged response to trastuzumab in a patient with HER2nonamplified breast cancer with elevated HER2 dimerization harboring an ERBB2 S310F mutation. J Natl Compr Canc Netw. 2015;13:1066-70.

26. Farmer H, McCabe N, Lord CJ, Tutt ANJ, Johnson DA, Richardson TB, et al. Targeting the DNA repair defect in BRCA mutant cells as a therapeutic strategy. Nature. 2005:434:917-21.

27. Bouwman $P$, Jonkers J. The effects of deregulated DNA damage signalling on cancer chemotherapy response and resistance. Nat Rev Cancer. 2012;12:587-98.

28. Bang YJ, Im SA, Lee KW, Cho JY, Song EK, Lee KH, et al. Randomized, doubleblind Phase II trial with prospective classification by ATM protein level to evaluate the efficacy and tolerability of olaparib plus paclitaxel in patients with recurrent or metastatic gastric cancer. J Clin Oncol. 2015;33:3858-65.

29. Bang YJ, Boku N, Chin KW, Lee SH, Park S, Qin SY, et al. Olaparib in combination with paclitaxel in patients with advanced gastric cancer who have progressed following first-line therapy: Phase III GOLD study. Ann Oncol. 2016;27(Suppl 6):vi552-87

30. Tsuchida N, Murugan AK, Grieco M. Kirsten Ras* oncogene: significance of its discovery in human cancer research. Oncotarget. 2016;7:46717-33.

31. Cox AD, Fesik SW, Kimmelman AC, Luo J, Der CJ. Drugging the undruggable RAS: mission possible? Nat Rev Drug Discov. 2014;13:828-51.

32. Rahman MT, Nakayama K, Rahman M, Katagiri H, Katagiri A, Ishibashi T, et al. KRAS and MAPK1 gene amplification in type II ovarian carcinomas. Int J Mol Sci. 2013:14:13748-62.

33. Blumenschein GR, Smit EF, Planchard D, Kim DW, Cadranel J, De Pas T, et al. A randomized phase $\|$ study of the MEK1/MEK2 inhibitor trametinib (GSK1120212) compared with docetaxel in KRAS-mutant advanced nonsmall-cell lung cancer (NSCLC). Ann Oncol. 2015;26:894-901.

34. Sherr CJ, Beach D, Shapiro Gl. Targeting CDK4 and CDK6: from discovery to therapy. Cancer Discov. 2016;6:353-67.

35. Wiedemeyer WR, Dunn IF, Quayle SN, Zhang J, Chheda MG, Dunn GP, et al. Pattern of retinoblastoma pathway inactivation dictates response to CDK4/6 inhibition in GBM. Proc Natl Acad Sci U S A. 2010;107:11501-6.

36. Konecny GE, Winterhoff B, Kolarova T, Qi J, Manivong K, Dering J, et al. Expression of p16 and retinoblastoma determines response to CDK4/6 inhibition in ovarian cancer. Clin Cancer Res. 2011;17:1591-602.

37. Young RJ, Waldeck K, Martin C, Foo JH, Cameron DP, Kirby L, et al. Loss of CDKN2A expression is a frequent event in primary invasive melanoma and correlates with sensitivity to the CDK4/6 inhibitor PD0332991 in melanoma cell lines. Pigment Cell Melanoma Res. 2014;27:590-600. 
38. Gao J, Adams RP, Swain SM. Does CDKN2A loss predict palbociclib benefit? Curr Oncol. 2015;22:e498-501.

39. Berns K, Horlings HM, Hennessy BT, Madiredjo M, Hijmans EM, Beelen K, et al. A functional genetic approach identifies the PI3K pathway as a major determinant of trastuzumab resistance in breast cancer. Cancer Cell. 2007; 12:395-402.

40. Mohd Sharial MS, Crown J, Hennessy BT. Overcoming resistance and restoring sensitivity to HER2-targeted therapies in breast cancer. Ann Oncol. 2012;23:3007-16.

41. Zuo Q, Liu J, Zhang J, Wu M, Guo L, Liao W. Development of trastuzumabresistant human gastric carcinoma cell lines and mechanisms of drug resistance. Sci Rep. 2015;5:11634.

42. Kelly CM, Janjigian YY. The genomics and therapeutics of HER2-positive gastric cancer-from trastuzumab and beyond. J Gastrointest Oncol. 2016;7:750-62.

43. Lee JY, Hong M, Kim ST, Park SH, Kang WK, Kim KM, et al. The impact of concomitant genomic alterations on treatment outcome for trastuzumab therapy in HER2-positive gastric cancer. Sci Rep. 2015:5:9289.

44. Alexandrov LB, Nik-Zainal S, Siu HC, Leung SY, Stratton MR. A mutational signature in gastric cancer suggests therapeutic strategies. Nat Commun. 2015;6:8683.

45. Sahasrabudhe R, Lott P, Bohorquez M, Toal T, Estrada AP, Suarez JJ, et al Germline mutations in PALB2, BRCA1, and RAD51C, which regulate DNA recombination repair, in patients with gastric cancer. Gastroenterology. 2017;152:983-986.e6.

46. Konstantinopoulos PA, Ceccaldi R, Shapiro Gl, D'Andrea AD. Homologous recombination deficiency: exploiting the fundamental vulnerability of ovarian cancer. Cancer Discov. 2015;5:1137-54.

47. Telli ML, Timms KM, Reid J, Hennessy B, Mills GB, Jensen KC, et al. Homologous recombination deficiency (HRD) score predicts response to platinum-containing neoadjuvant chemotherapy in patients with triplenegative breast cancer. Clin Cancer Res. 2016;22:3764-73.

48. Lee YS, Cho YS, Lee GK, Lee S, Kim YW, Jho S, et al. Genomic profile analysis of diffuse-type gastric cancers. Genome Biol. 2014;15:R55.

\section{Submit your next manuscript to BioMed Central} and we will help you at every step:

- We accept pre-submission inquiries

- Our selector tool helps you to find the most relevant journal

- We provide round the clock customer support

- Convenient online submission

- Thorough peer review

- Inclusion in PubMed and all major indexing services

- Maximum visibility for your research

Submit your manuscript at www.biomedcentral.com/submit
Biomed Central 